\title{
PAEDIATRIC RETINOBLASTOMA PRESENTATION IN A REGIONAL CANCER CENTRE IN PAKISTAN
}

\author{
Saadiya J. Khan Inayat \\ Paediatric Oncology Department, Shaukat Khanum Cancer Hospital and Research Centre, Lahore, Pakistan \\ Received: 1 March 2017 / Accepted: 18 June 2017
}

\begin{abstract}
Purpose: Retinoblastoma (RB) is a common paediatric intraocular tumour. In the developed world, RB accounts for $<5 \%$ of all paediatric cancers. In the developing world, RB may comprise about $10-15 \%$ of all diagnosed paediatric cancers according to hospital-based retrospective study in India.

Methods: Medical records for all diagnoses of RB at our regional cancer centre were retrospectively reviewed from January 2005 to September 2015 after IRB approval.

Results: A total of $258(100 \%)$ patient charts were reviewed. More male $n=163(63.2 \%)$ patients were seen than females $n=95$ (36.8\%). Most patients were diagnosed before the age of 5 years. Positive family history was seen in $20(7.8 \%)$ patients. The total number of bilateral disease was seen in $75(29.1 \%)$ patients and $183(70.9 \%)$ had unilateral diagnoses. Median age at presentation was 24 months for bilateral and 36 months for unilateral disease.

Conclusions: The median age at presentation shows a significant delay in the establishment of diagnosis for both unilateral and bilateral RB. Compared to western data, there were a higher number of male patients seen. Financial constraints, access to primary health care, lack of trained personnel and cultural practices are likely factors, leading to delayed presentation and advanced disease state.
\end{abstract}

Key words: Retinoblastoma, ocular tumours, leukocoria

\section{Introduction}

Retinoblastoma (RB) is the most common intraocular tumour of childhood. It is associated with the RB gene mutation. When congenital it is present at birth and clinically picked up either during well-child checks or at an early age by parents. In cases where the disease is sporadic or non-hereditary, it presents in a single eye later in life between 3 and 5 years of age. Two-thirds of all cases of RB are diagnosed before the age of 2 years while $95 \%$ of cases are diagnosed before 5 years of age. ${ }^{[1]}$

The incidence of $\mathrm{RB}$ is considered to be constant around the world at one case per 15,000-20,000 live births, which corresponds to about 9000 new cases every year. ${ }^{[2,3]}$ Management of RB has improved over the years. Today, in the developed world, the early presentation of disease, access

Correspondence: Dr. Saadiya J. Khan, Department of Paediatric Oncology, Shaukat Khanum Cancer Hospital and Research Centre, Lahore, Pakistan. Email: saadiyajaved@skm.org.pk to care and advancement in therapy have led to a survival rate of $>90 \%{ }^{[4,5]}$ In developing countries, these rates are lower secondary to different reasons and an important one being access to health-care resulting in advanced disease at the time of presentation to the oncology service. ${ }^{[6,7]}$

In the 1950s, the Reese-Ellsworth classification system was developed to predict the prognosis after treatment with radiation. Now, clinicians are using the International Classification of RB (ICRB) to better predict outcomes of intraocular RB without the need for external beam radiation. The ICRB grading is as follows:

Group A: Small intraretinal tumours ( $<3 \mathrm{~mm})$ away from foveola and disc.

Group B: Tumours $>3 \mathrm{~mm}$, macular or juxtapapillary location, or with subretinal fluid.

Group C: Tumour with focal subretinal or vitreous seeding within $3 \mathrm{~mm}$ of tumour.

Group D: Tumour with diffuse subretinal or vitreous seeding $>3 \mathrm{~mm}$ from tumour. 
Group E: Extensive RB occupying $>50 \%$ of the globe with or without neovascular glaucoma, haemorrhage and extension of tumour to optic nerve or anterior chamber.

Disease presentation is varied and can include leukocoria, strabismus, proptosis, redness and so on. These symptoms can overlap with other ophthalmologic conditions. This can delay diagnosis by primary health-care providers in developing countries not familiar with $\mathrm{RB}$, a rare but common cancer in the eyes of young children. The aim of this study is to present our institutional experience of treatment and outcome in children with $\mathrm{RB}$ seen at a large freestanding cancer hospital in Lahore, Pakistan.

\section{Methods}

We conducted a retrospective chart review of all patients diagnosed with $\mathrm{RB}$ at a large regional cancer centre in Pakistan between January 2005 and September 2015. These patients were accepted into the system after a detailed history and examination. Studies done before starting treatment included ocular ultrasonography, examination under anaesthesia, computed tomography or a magnetic resonance imaging, bilateral bone marrow biopsy, lumbar puncture, audiometry and creatinine clearance. The data collection for this analysis included age at presentation, sex, family history of RB, clinical presentation, treatment administered, treatment compliance and outcome duration of survival.

\section{Results}

A total of $258(100 \%)$ patients were treated during the study period. Of these, $163(63.2 \%)$ were boys and 95 (36.8\%) girls [Table 1]. Chart review showed $183(70.9 \%)$ patients had unilateral disease while 75 (29.1\%) had bilateral disease. The median age of diagnosis for bilateral disease was 24 months (range, 2-96 months) and 36 months (range, 1-132 months) for unilateral disease. $>50 \%$ of the patients were older than 24 months and the oldest age at presentation was 11 years.

There were $20(7.8 \%)$ families who reported a positive family history for RB. 10 (50\%) of these had unilateral disease and $10(50 \%)$ had bilateral disease. The most common presenting symptoms were leukocoria $n=145(56.2 \%)$ and proptosis $n=49$ (18.9\%). Chart
Table 1: Patient characteristics

\begin{tabular}{l|c}
\hline Signs and symptoms & $\boldsymbol{n}(\%)$ \\
\hline Leukocoria & $146(56.6)$ \\
\hline Proptosis & $49(19)$ \\
\hline Extraocular mass & $27(10.5)$ \\
\hline Vision loss & $14(5.4)$ \\
\hline Strabismus & $12(4.7)$ \\
\hline Others & $10(3.9)$ \\
\hline M: F ratio & $1.7: 1$ \\
\hline
\end{tabular}

review showed that $108(41.8 \%)$ patients were lost to follow-up, 47 (18.2\%) died and 103 (39.9\%) were alive at the last follow-up at 2 years. $196(75.9 \%)$ patients were treated surgically with an enucleation or exenteration, whereas the rest of the $62(24 \%)$ were those that were managed with chemotherapy without surgical intervention at our institution. Specimens for $163(63.2 \%)$ patients were available for pathological review.

Our patients received chemotherapy consisting mainly of carboplatin, vincristine, etoposide, cyclophosphamide and doxorubicin in addition to enucleation. No facilities for cryotherapy, brachytherapy, intravitreal or intra-arterial chemotherapy were available to our patients at that time. A dedicated genetic counselling service was also not accessible to patients.

\section{Discussion}

We have presented a freestanding cancer centre's data over a significantly long period of time in Pakistan. There is a substantial amount of illiteracy, unfamiliarity and lack of awareness to seek treatment for paediatric cancers in our setting. Some of the families that do sense a problem resort to alternative medicine before realizing the need to pursue oncologic care. There is no sex predilection in $\mathrm{RB}$, yet we see a male predominance. The patriarchal society that exists in our setup can be a reason for this observation. ${ }^{[8]}$

In the developed world, patients present at an early age with a multidisciplinary approach formulated at the onset of therapy. This results in remarkable survival outcome along with vision preservation and reduction in enucleation rates. Treatment compliance and follow-up are also better with early presentation of disease. 
Follow-up of our patients was inadequate with very high rates of treatment abandonment. This is partly due to resource-limited setting and potential lifesaving yet surgically disfiguring options put forward in cases of extraocular disease.

One of the other key issues this study highlights is the diagnosis and treatment of paediatric patients with $\mathrm{RB}$. The age at presentation is significantly more when compared to western data ${ }^{[8-10]}$ subsequently resulting in more advanced disease at the time of presentation with mostly Group $\mathrm{C}$ to $\mathrm{E}$ eyes. When there is such progressive disease, the primary objective is to save the patient's life. Therapeutic options for globe and vision salvage sans enucleation or exenteration are very few. ${ }^{[11,12]}$

There are institutions in the developed world such as the Childhood Eye Cancer Trust and others that promote the use of smartphones in detecting a red reflex or the presence of a white reflex. The white reflex can be a sign of something more serious such as RB. Nowadays, there are smartphone applications that help parents and families detect a white reflex. These applications enable parents detect a white reflex by clicking pictures of their children.

Review of our institutional experience has led us to ascertain our practice limitations and consequently suggest areas of improvement. First, we need to educate families about the signs and symptoms of RB. With the widespread availability of smartphones, there is a need to educate masses about the use of these devices for the detection of a white reflex. The understanding to seek cure when children have low-risk disease will result in better rates of ocular salvage and sight preservation. ${ }^{[13]}$ As per the International Intraocular RB Classification, Group A to $\mathrm{C}$ eyes are considered amenable to early intervention. Primary care providers at the district level in our setup should also be trained to recognize such oncologic cases earlier and initiate timely referrals. Parents of newborns should be advised to get routine eye checkups from primary health-care providers.

The second tier of struggle for patients was a gap in diagnostic skills and treatment consensus between medical care providers and their respective facilities. With the development of our freestanding cancer hospital, we have implemented a uniform staging workup including pathology review and standard treatment guidelines. We recommend organizing workshops and teaching conferences to help train physicians from other institutions who are not familiar with this paediatric diagnosis yet tend to see these patients in their practice.

Our team comprises members from paediatric oncology, ophthalmology, pathology and radiology responsible for discussing new and existing patients with a diagnosis of $\mathrm{RB}$ in a multidisciplinary meeting.

\section{Conclusions}

We encourage our local colleagues to reach out to skilled and highly specialized medical professionals for any queries about paediatric RB. Our institutional agenda is to propagate early detection and attempt optimal local tumour control with focal consolidative procedures such as laser photocoagulation, cryotherapy and local chemotherapy. We are working to introduce intravitreal and intra-arterial chemotherapy where possible ${ }^{[14]}$ in early disease.

\section{Conflict of Interest}

The authors declare that they have no conflict of interest.

\section{References}

1. Ries LA, Smith MA, Gurney JG. Cancer incidence and survival among children and adolescents: United States SEER Program 1975-1995. Bethesda, MD: National Cancer Institute, SEER Program; 1999. NIH Pub. No. 99-4649.

2. Kivelä $\mathrm{T}$. The epidemiological challenge of the most frequent eye cancer: Retinoblastoma, an issue of birth and death. Br J Ophthalmol 2009;93:1129-31.

3. Shields JA, Shields CL. Management of retinoblastoma. In: Shields JA, Shields CL. Intraocular Tumours. An Atlas and Textbook. Philadelphia, PA: Lippincott Williams Wilkins; 2008. p. 334-51.

4. Berman EL, Donaldson CE, Giblin M, et al. Outcomes in retinoblastoma, 1974-2005: The children's hospital, westmead. Clin Exp Ophthalmol 2007;35:5-12.

5. MacCarthy A, Birch JM, Draper GJ, et al. Retinoblastoma: Treatment and survival in great Britain 1963 to 2002. Br J Ophthalmol 2009;93:38-9.

6. Bowman RJ, Mafwiri M, Luthert P, et al. Outcome of retinoblastoma in East Africa. Pediatr Blood Cancer 2008;50:160-2.

7. Bhurgri Y, Muzaffar S, Ahmed R, et al. Retinoblastoma in Karachi, Pakistan. Asian Pac J Cancer Prev 2004;5:159-63.

8. Meel R, Radhakrishnan V, Bakhshi S. Current therapy and 
recent advances in the management of retinoblastoma. Indian J Med Paediatr Oncol 2012;33:80-8.

9. Aerts I, Lumbroso-Le Rouic L, Gauthier-Villars M, et al. Retinoblastoma. Orphanet J Rare Dis 2006;1:31.

10. Abramson DH, Frank CM, Susman M, et al. Presenting signs of retinoblastoma. J Pediatr 1998;132:505-8.

11. Chantada G, Fandiño A, Manzitti J, et al. Late diagnosis of retinoblastoma in a developing country. Arch Dis Child 1999;80:171-4.
12. Rodriguez-Galindo C, Wilson MW, Chantada G, et al. Retinoblastoma: One world, one vision. Pediatrics 2008;122:e763-70.

13. Leander C, Fu LC, Peña A, et al. Impact of an education program on late diagnosis of retinoblastoma in Honduras. Pediatr Blood Cancer 2007;49:817-9.

14. Selistre SG, Maestri MK, Santos-Silva $\mathrm{P}$, et al. Retinoblastoma in a pediatric oncology reference center in Southern Brazil. BMC Pediatr 2016;16:48. 